\title{
A STUDY OF THE GENOTYPE BY ENVIRONMENT INTERACTION SHOWN BY GERMINATING SEEDS OF BRASSICA NAPUS
}

\author{
J. R. WITCOMBE* and W. J. WHITTINGTON \\ Nottingham University School of Agriculture, Sutton Bonington \\ Loughborough, Leicestershire
}

Received 6.vi.70

\section{INTRODUCTION}

INCREASING interest is being shown in the variability between plants for genotype by environment interactions and a number of methods have been proposed to analyse this variation. The methods of Finlay and Wilkinson (1963) and Perkins and Jinks (1968) which are similar to that proposed earlier by Yates and Cochran (1938) are regression analyses in which the average yield of all lines grown in each environment are used to evaluate

TABLE 1

The parameters used in the methods of Perkins and Finks (1968) and Yates and Cochran (1938) or Finlay and Wilkinson (1963) and their interrelationships

Yates and Cochran (1938)

Finlay and Wilkinson (1963) Perkins and Jinks (1968)

Parameter

Slope

Genetic value

Grand mean

Environment

$$
\text { Statistic }
$$

\section{$b$}

$\bar{y}$

$\overline{\bar{y}}$

$x$

$$
\text { Statistic }
$$

$\beta$

$d$

$\mu$

$\varepsilon$
Relationship

$b-1=\beta$

$\bar{y}-\mu=d$

$\overline{\bar{y}}=\mu$

$x-\mu=\varepsilon$

that environment, and the response of each genotype to the range o environments is assessed by its regression coefficient. The average response of all the genotypes has a regression coefficient of one or zero depending on the analysis used, and the response of individual genotypes can be assessed relative to this mean response.

The behaviour of any genotype is described by its regression coefficient and a measure of its yield which will account for its genetic value. Yield can be assessed in one environment (the $y$ values of the regresson analyses) or as the average yield $(\bar{y})$. A scatter diagram can be plotted with $b$ and $y$ values as co-ordinates and this allows, for example, superior genotypes for both parameters to be recognised.

The parameters used in the methods of Yates and Cochran (1938) and Finlay and Wilkinson (1963) or Perkins and Jinks (1968) are simply related (table 1 ).

The analytical methods proposed have been tested against experimental data from field experiments in which little was known about the physical nature of the environmental circumstances. In the experiments to be reported, however, the germination rates of half-sib families of cultivated rape (Brassica napus L.) were studied in controlled environments differing for temperature and water potential.

* School of Plant Biology, University College of North Wales, Bangor. 
The experiments were not designed with the specific objective of investigating environmental effects on germination, but instead aimed to provide results which allowed a study of the effects of known environments either separately or combined together for statistical analysis. The results enable certain conclusions to be drawn which aid the interpretation of experiments in which environmental variables are known but unmeasured or are entirely unknown.

\section{Materials and methods}

The experiments were carried out on seeds collected from 45 plants selected at random from an open-pollinated population of spring oil seed

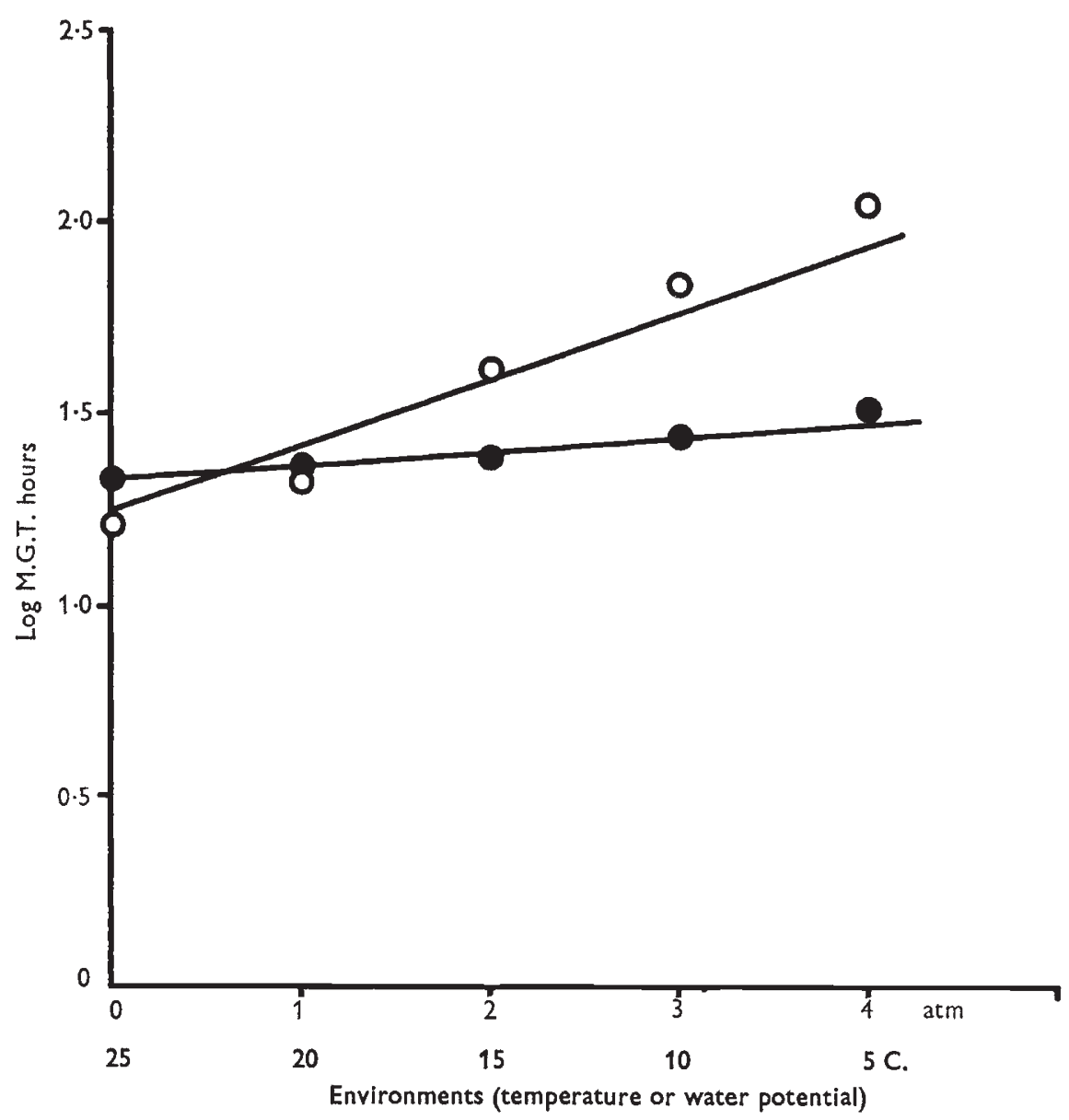

FIG. 1.-Linear regression $\left(b=0.21^{* *}\right.$ and $\left.0.04^{* *}\right)$ of $\log _{10}$ mean time to germination (M.G.T. hours) over 45 genotypes in various temperature $\left({ }^{\circ}\right)$ and water-potential environments (๑).

rape var. Zollerngold. The seeds from each of the plants were retained separately to form 45 half-sib families in which the members of each family had a common maternal parent. As germination characteristics are markedly influenced by the maternal parent, the pollen parents are of 
less importance than in normal half-sib families, with the result that these families will be genetically more distinct. Although the relationships between the families cannot, therefore, be precisely defined, they may be held to be distinct genotypes and thus the families for convenience are referred to in the paper as genotypes.

The seeds were germinated in ten different environments; $0,1,2,3,4$ atmospheres water potential at $20^{\circ} \mathrm{C}$. and at $5^{\circ}, 10^{\circ}, 15^{\circ}, 20^{\circ}$ and $25^{\circ} \mathrm{C}$. at zero atmospheres water potential. These environments are later referred to as water-potential environments and temperature environments respectively. Solutions of differing water potential were prepared by using polyethylene glycol (Carbowax M.W. 1540, which was purified by passing it

TABLE 2

Separate and combined regression analyses of variance of $\log _{10}$ mean time to germination for 45 genotypes in five different water-potential and temperature environments

\begin{tabular}{|c|c|c|c|c|c|c|c|c|c|}
\hline \multirow[b]{2}{*}{ Item } & \multicolumn{3}{|c|}{$\begin{array}{l}\text { Water-potential } \\
\text { environments }\end{array}$} & \multicolumn{3}{|c|}{ Temperature environments } & \multicolumn{3}{|c|}{ Combined analysis } \\
\hline & d.f. & M.S. & V.R. & d.f. & M.S. & V.R. & d.f. & M.S. & V.R. \\
\hline Environments & 4 & 0.2307 & $1281 \cdot 4 * * *$ & 4 & 4.8059 & $17475 \cdot 7 * * *$ & 9 & $2 \cdot 6492$ & $6461 \cdot 3 * * *$ \\
\hline Genotypes & 44 & 0.0088 & $48 \cdot 7 * * *$ & 44 & 0.0077 & $5 \cdot 4 * * *$ & 44 & 0.0152 & $37 \cdot 1 * * *$ \\
\hline $\begin{array}{l}\text { Heterogeneity } \\
\text { between } \\
\text { regressions }\end{array}$ & 44 & 0.0010 & $5 \cdot 2 * * *$ & 44 & 0.0014 & $5 \cdot 1 * * *$ & 44 & 0.0018 & $4 \cdot 2 * * *$ \\
\hline Remainder & 132 & 0.0002 & 0.4 & 132 & 0.0003 & 0.5 & 352 & 0.0004 & $0 \cdot 8$ \\
\hline Error & 225 & 0.0005 & - & 225 & 0.0005 & - & 510 & 0.0005 & - \\
\hline
\end{tabular}

in aqueous solution through both positive and negative ion exchange columns. The Carbowax solutions were made up to concentrations which gave the required water potential from calibrations determined with a thermocouple psychrometer. Ten seeds were germinated in plastic petri dishes $(51 \mathrm{~mm}$. diameter) on Whatman's No. 1 filter paper. In the temperature environments there was $0.75 \mathrm{ml}$. of distilled water per dish, and in the water-potential environments $1 \mathrm{ml}$. of solution or distilled water per dish. There were two replicates of each genotype in each treatment.

The germination of the seeds, as emergence of the radicle, was recorded at equally spaced time intervals and about 15 successive readings were made in every environment.

The germination rates of the genotypes have been expressed as mean germination time in hours (M.G.T.) where this is the average time for the seeds which germinated in a sample to do so. Mean germination times were logged to achieve regressions that were linear. For clarity in the text M.G.T. values are referred to as germination rates.

\section{REsults}

\section{(a) The extent and significance of genetic variability}

In every environment over 98 per cent. of the seeds germinated and thus the effects were solely on the rates at which the seeds germinated. Low temperature delayed germination far more than the water-potential treatments (fig. 1). 
Analyses for the results from the environments separately or combined together showed that the genotype effects and linear genotype environment

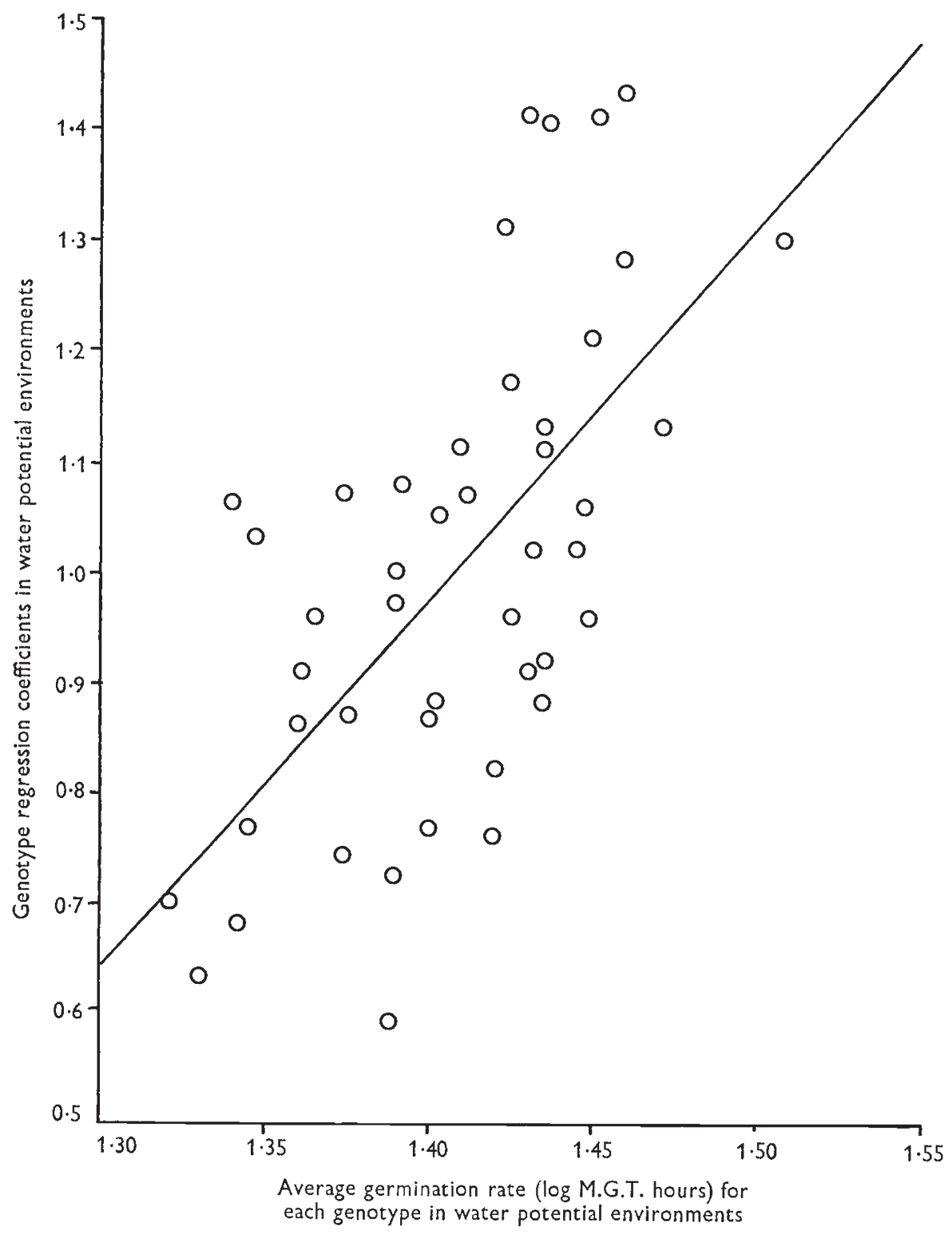

FIG. 2a.-Linear regression $\left(b=3 \cdot 36^{* * *}\right)$ of genotype regression coefficients in the waterpotential environments against average germination rate $\left(\log _{10}\right.$ M.G.T. hours) of each genotype in the water-potential environments.

interactions were highly significant. The environmental components, as would be expected from the wide range of treatments imposed, were very large (table 2). Identical mean squares are obtained from the Finlay and Wilkinson (1963) analysis or the Perkins and Jinks (1968) analysis. 
(b) The characteristics of the genetic variability in the different environmental systems

Scatter diagrams of regression coefficients against average germination rates for the water potential and temperature environments (figs. $2 a$ and $2 b$ )

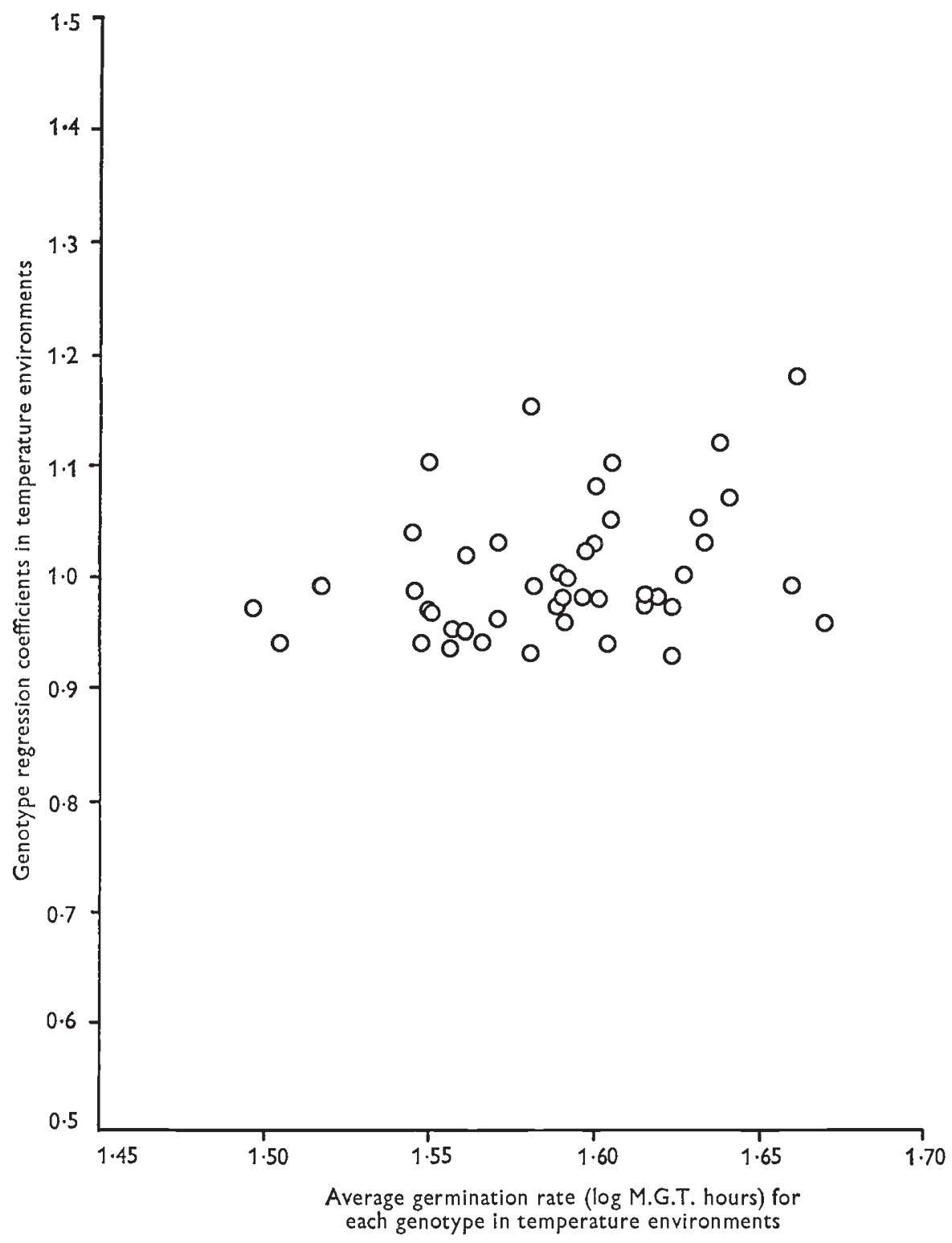

FIG. $2 b$. - Linear regression $(b=0 \cdot 19$ N.S.) of genotype regression coefficients in temperature environments against average germination rate ( $\log _{10}$ M.G.T. hours) of each genotype in the temperature environments.

showed similar variability for average germination rates $(V[d])$. The variability for regression coefficients $(V[g])$ was, however, much larger in the water-potential than in the temperature environments. If these pro- 


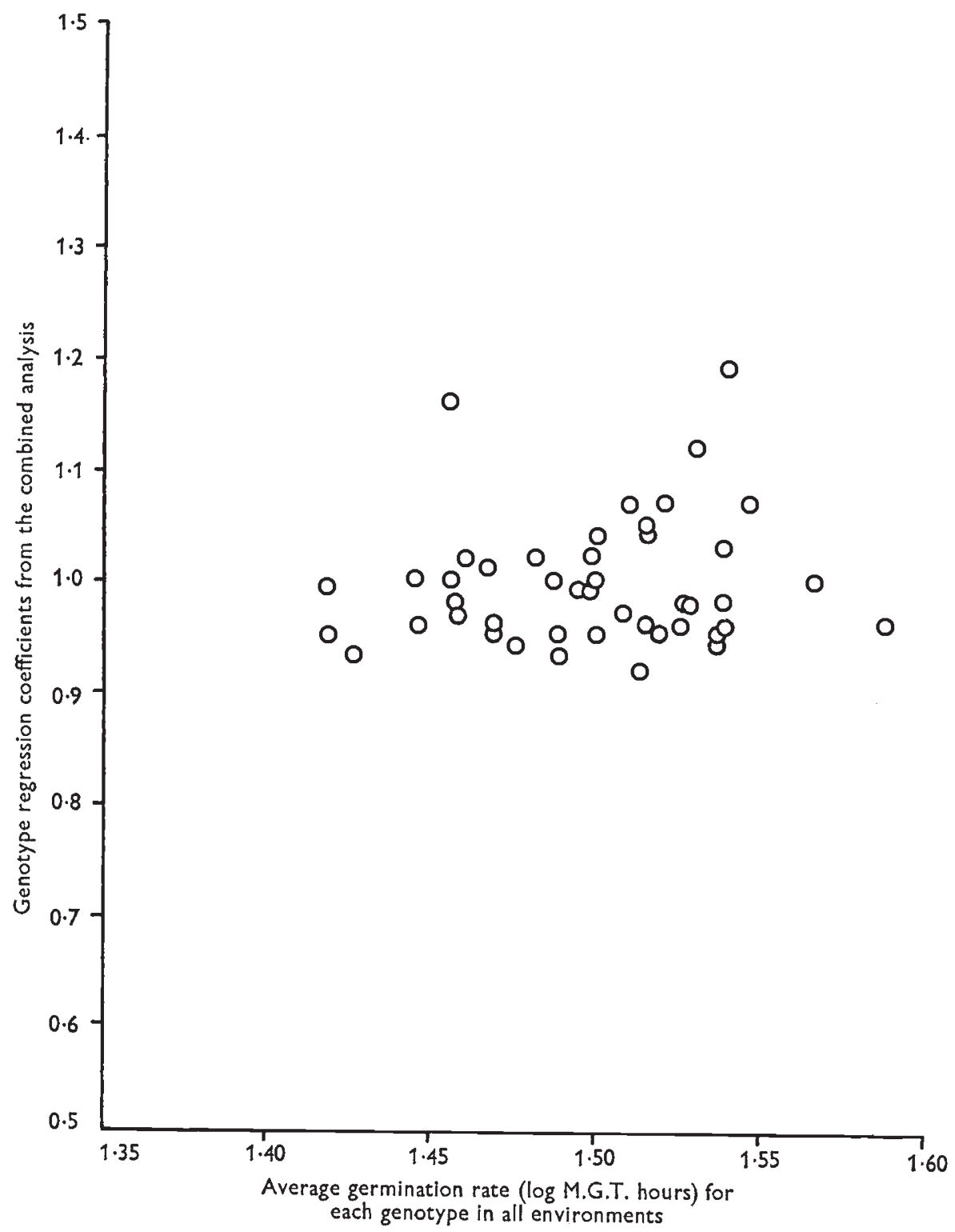

FIG. $2 c$. - Linear regression $(0.26 \mathrm{~N}$.S. $)$ of genotype regression coefficients from the combined analyses against average germination rate $\left(\log _{10}\right.$ M.G.T. hours) for each genotype in all environments.

\section{TABLE 3}

Percentage of the total genetic variability attributable to linear genotype by environment interactions

Genetic variability as

Environmental system

Temperature analysis

Water analysis

Combined analysis

$$
\begin{gathered}
\frac{V[g]}{V[d]+V[g]} \times 100 \\
15 \cdot 6 \\
9 \cdot 8 \\
10 \cdot 3
\end{gathered}
$$


portions are examined from the regression analyses instead of the scatter diagrams the situation is reversed (table 3 ). This anomaly was due to the proportionately larger remainder mean square in the water-potential analyses. This meant that the variability for regression coefficients contributed less to the total mean square in this analysis than might have been expected from the scatter diagram.

The scatter diagrams for the combined and temperature analyses were similar (figs. $2 b$ and $2 c$ ). The similarity was due to the inclusion of the larger values from the temperature treatments relative to those from the

TABLE 4

Correlation of bw and bt values with the germination rates of the water potential environments and temperature environments respectively

\begin{tabular}{|c|c|c|c|}
\hline $\begin{array}{c}\text { Water } \\
\text { environment }\end{array}$ & $\begin{array}{l}\text { Regression } \\
\text { coefficients }\end{array}$ & $\begin{array}{l}\text { Correlation } \\
\text { coefficients }\end{array}$ & $\begin{array}{l}\text { Significance of } \\
\text { regression }\end{array}$ \\
\hline $\begin{array}{l}0 \mathrm{~atm} \\
1 \mathrm{~atm} \\
2 \mathrm{~atm} \\
3 \mathrm{~atm} \\
4 \mathrm{~atm}\end{array}$ & $\begin{array}{l}+1.97 \pm 0.86 \\
+2.97 \pm 0.76 \\
+2.95 \pm 0.62 \\
+3.37 \pm 0.59 \\
+2.86 \pm 0.27\end{array}$ & $\begin{array}{l}0 \cdot 33 \pm 0 \cdot 15 \\
0 \cdot 51 \pm 0 \cdot 13 \\
0 \cdot 58 \pm 0 \cdot 10 \\
0 \cdot 66 \pm 0 \cdot 11 \\
0 \cdot 85 \pm 0 \cdot 04\end{array}$ & $\begin{array}{l}* \\
* * * \\
* * * \\
* * * \\
* * *\end{array}$ \\
\hline Average & $+3 \cdot 36 \pm 0.59$ & $0.65 \pm 0 \cdot 11$ & $* * *$ \\
\hline \multicolumn{4}{|l|}{$\begin{array}{l}\text { Temperature } \\
\text { environment }\end{array}$} \\
\hline $\begin{array}{l}25^{\circ} \mathrm{C} \text {. } \\
20^{\circ} \mathrm{C} . \\
15^{\circ} \mathrm{C} . \\
10^{\circ} \mathrm{C} . \\
5^{\circ} \mathrm{C} \text {. }\end{array}$ & $\begin{array}{r}-0.84 \pm 0.38 \\
-0.43 \pm 0.40 \\
0 \cdot 25 \pm 0.34 \\
0.32 \pm 0.30 \\
0.73 \pm 0.27\end{array}$ & $\begin{array}{l}0 \cdot 31 \pm 0 \cdot 15 \\
0 \cdot 16 \pm 0 \cdot 15 \\
0 \cdot 11 \pm 0 \cdot 15 \\
0 \cdot 16 \pm 0 \cdot 15 \\
0 \cdot 38 \pm 0 \cdot 14\end{array}$ & $\begin{array}{l}* \\
\text { N.S. } \\
\text { N.S. } \\
\text { N.S. } \\
* *\end{array}$ \\
\hline ige & $0 \cdot 19 \pm 0 \cdot 39$ & $0.08 \pm 0 \cdot 15$ & N.S. \\
\hline
\end{tabular}

$*, * *, * * *$ indicate significance of the regression coefficient at $\mathrm{P}=0.05,0.01$ and 0.001 respectively.

water-potential environments into the combined analysis. The distribution of points in the scatter diagram for the water-potential environments differed markedly from the other two distributions in that the regression coefficients and germination rates had a significant linear relationship. The pattern of genetic variability thus altered when the environmental regimes imposed were physically different.

\section{(c) The correlation of regression coefficients with germination rates}

The regression coefficients were also correlated to the individual values that made up the average germination rates. Thus, the regression coefficients of genotypes in the water-potential environments (bw values), where a significant correlation between regression coefficients and average germination rates was found, were correlated to the germination rates in each of the five water-potential environments separately. The correlation improved as the environment considered produced higher values for germination rates (table 4). The correlation of regression coefficients with the germination rates in the four atmospheres water-potential environment was thus greater than the average.

When the temperature environments were considered the regression 
coefficients ( $b t$ values) and the germination rates varied from negative and significant to positive and significant as the $y$ values became higher (table 4). Thus the correlations between $b$ and $y$ values are dependent on the physical nature of the environment and on the particular range of the environments from which the $y$ values are taken.

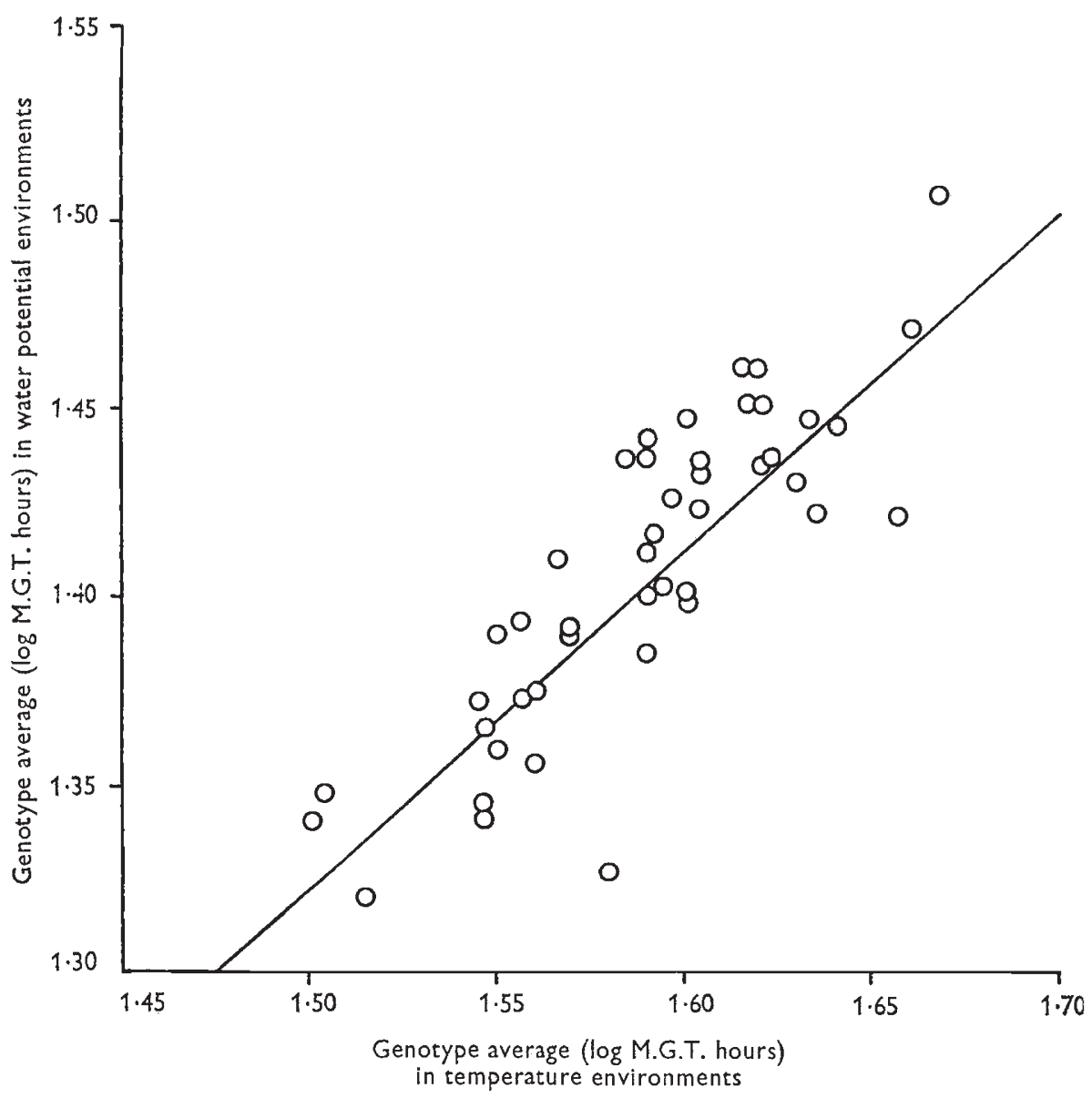

FIG. 3.-Linear regression $\left(0 \cdot 83^{* * *}\right)$ of genotype average germination rates $\left(\log _{10}\right.$ M.G.T. hours) in water-potential environment against genotype average germination rates ( $\log _{10}$ M.G.T. hours) in temperature environments.

(d) The specificity of the regression coefficients and average germination rates

The possibility that genotypes had regression coefficients that were common to more than one environmental system was examined, but the regression of the coefficients from the temperature environments on those from the water-potential environments was not significant. The regression coefficients attributable to each genotype in the water-potential and temperature environments were also compared and the regression coefficients of 12 families were found to differ significantly between these environments. This number of significant comparisons is more than would be expected by chance at the 5 per cent. level of probability. 
A significant correlation was found to exist between the average germination rates in the temperature and water-potential systems (fig. 3). Thus an early germinating genotype germinated early in both environmental systems, but its response did not necessarily remain the same. The correlation between average germination rates would not necessarily apply over other environmental ranges since significant interaction effects were present.

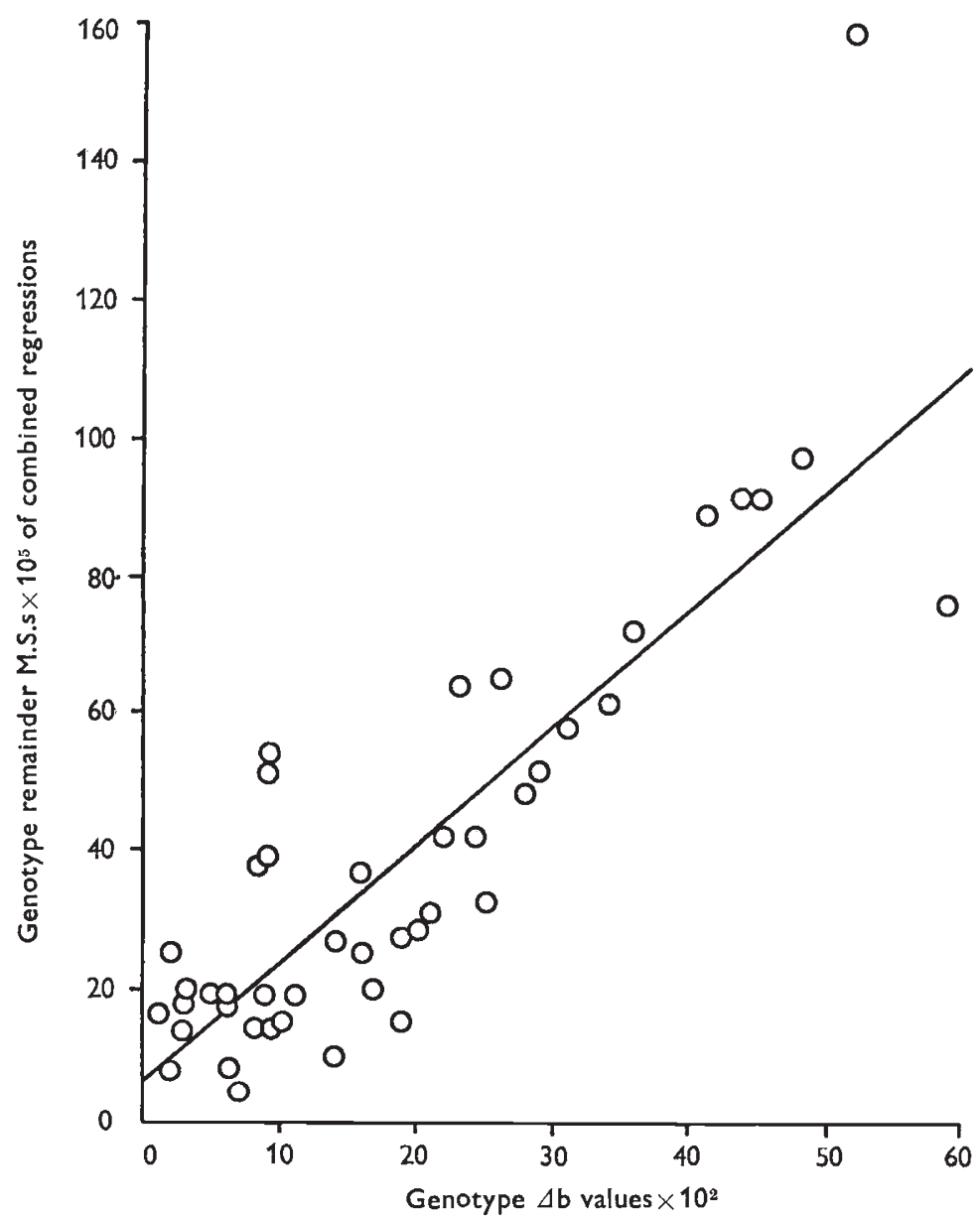

FIG. 4. - Linear regression $\left(b=2 \cdot 84^{* * *}\right)$ of genotype remainder mean squares (M.S. $\left.\times 10^{4}\right)$ of combined regressions against the difference $\left(\times 10^{2}\right)$ between the genotype regression coefficients in the temperature and water potential environments ( $\Delta b$ values).

(e) The change in genotype behaviour in the two environmental systems and the stability of response

The change in response of each genotype to the two environmental systems can be calculated as the difference $(\Delta b)$ between the two regression coefficients, i.e. $\Delta b=b t-b w$, where $\Delta b$ is assigned a positive value. The $\Delta b$ value is a measure of the uniformity of response of a genotype to the two physical parameters. Plotting $\Delta b$ values for each genotype against the remainder terms of the combined analyses for each genotype showed a significant relationship (fig. 4). Thus if a genotype has a small $\Delta b$ valu 
(i.e. a uniform response) then a combined regression of the data for that genotype from the two environmental systems will have a small remainder term. The opposite applies to a genotype with a large $\Delta b$ value, i.e. a genotype which has a non-uniform response to the two environmental systems. Such a relationship is obviously a statistical truism since pooling regressions with similar coefficients and intercepts will result in a better fit than where two dissimilar regressions are pooled. But remainder terms should not be considered as merely error terms due to unpredictable processes, they are partly determined by the genotypes' change in response to temperature or water potential as the environment variable.

$\Delta b$ values were also significantly correlated to the remainder M.S.'s of the regressions calculated for the response of genotypes to the temperature and water-potential environments separately (table 5). This was almost

\section{TABLE 5}

Correlation of $\triangle \mathrm{b}$ and $\mathrm{b}$ values with the remainder M.S. of the three regression analyses

\section{Regression between}

$\Delta b /$ combined Rem. M.S.

$\Delta b /$ temperature Rem. M.S.

$\Delta b /$ water potential Rem. M.S.

$b t /$ combined Rem. M.S.

$b t /$ temperature Rem. M.S.

$b t /$ water potential Rem. M.S.

bws/combined Rem. M.S.

bws/temperature Rem. M.S.

bws/water potential Rem. M.S.

\begin{tabular}{|c|}
\hline $\begin{array}{l}\text { Regression } \\
\text { coefficient }\end{array}$ \\
\hline $\begin{array}{l}1.81 \pm 0.16 \\
0.49 \pm 0.35 \\
0.26 \pm 0.12\end{array}$ \\
\hline $\begin{array}{l}1 \cdot 33 \pm 0.80 \\
1 \cdot 09 \pm 0.52 \\
0.03 \pm 0.33\end{array}$ \\
\hline $\begin{array}{l}0.15 \pm 0.21 \\
0.07 \pm 0.15 \\
0.04 \pm 0.09\end{array}$ \\
\hline
\end{tabular}

\section{Correlation coefficient}

$0.86 \pm 0.04$

$0 \cdot 36 \pm 0 \cdot 14$

$0 \cdot 31 \pm 0 \cdot 15$

V.R.

$125 \cdot 20$

$6 \cdot 32$

$4 \cdot 75$

$0 \cdot 25 \pm 0 \cdot 13 \quad 2 \cdot 78$

$0 \cdot 31 \pm 0.15$

$0.02 \pm 0.15$

\section{$4 \cdot 43$}

0.01

\section{$0 \cdot 11 \pm 0 \cdot 15$}

$0.07 \pm 0.15$

$0 \cdot 07 \pm 0 \cdot 15$

0.46

$0 \cdot 21$

$0 \cdot 24$

$*, * *, * * *$ indicates significance of the regression coefficient at $\mathrm{P}=0.05,0.01$ and 0.001 respectively.

certainly due to these two environmental ranges also differing for the other environmental parameter. Effectively, for a genotype with a large $\Delta b$ value, two dissimilar responses are being plotted together. The situation is thus analogous to the pooling of the data for the temperature and water potential environments into a combined regression and it was seen that this produced remainder M.S.'s which were correlated with the $\Delta b$ values.

Correlations between regression coefficients values and remainder M.S.'s were also determined (table 5). Only the correlation between bt values and temperature regression analysis remainder M.S.'s was significant. There must have been within environment temperature variation to produce this correlation. It would be expected that the genotype with large responses to temperature would be most affected by this variation and have the largest deviation from a fitted regression line.

\section{$(f)$ The within environment within genotype variation and the $\mathrm{b}$ and $\Delta \mathrm{b}$ values}

The within environment within genotype variation (termed $e$ ) was assessed for all genotypes in each of the ten environments. The $e$ value was defined as the average of the two within replicate variances of a 
genotype and this value thus excluded the between replicate variation. It is interesting to see whether the phenotypic micro-environmental variation is related to the genotype regression coefficients. Accordingly the correlation of the $e$ values were determined for each environment with the $b t, b w$ and $\Delta b$ values as well as with the sums of the $b t$ and $b w$ values ( $b$ sum) (table 6 ).

Significant correlation were found that were in a marked pattern. In the low-temperature environments the $e$ values showed a correlation to the $b t$ values whereas in the high water stress environments the correlation was to the bw values. The variability is correlated to the regression coefficients: of the genotypes due to the fact that genotypes with high regression coefficients were more unstable and responded more to micro-environmental variation. From the pattern of correlations it can be inferred that the within

\section{TABLE 6}

The significance of the correlation of e values with the genotype regression coefficients, the $\Delta \mathrm{b}$ values and the $\mathrm{b}$ sum values

$\begin{gathered}\text { Environment to which } \\ e \text { values were related }\end{gathered}$
$\begin{gathered}5^{\circ} \mathrm{C} . \\ 10^{\circ} \mathrm{C} .\end{gathered}$

replicate variation was not homogeneous over the environments. There was probably variation for temperature in the low-temperature environments and variation for water potential in the high water potential environments.

The $\Delta b$ values did not show a correlation to the $e$ values. A positive correlation would not be expected to be present since an unstable genotype with both high $b t$ and $b w$ values would have a low $\Delta b$ value.

The sum of the $b t$ and $b w$ values should be the best indicators of within replicate within genotypic instability if there is variability for both water potential and temperature within the replicates. A genotype with a high response to both temperature and water potential would produce a high $e$ value under these conditions. However, the $b$ sum values only showed the same correlations to $e$ as the $b w$ values. This was due to two reasons. First, there was a marked correlation between $b$ sum values and $b w$ values; $b$ sum was the result of adding two sets of values of unequal variance and was largely determined by the bw values which had the highest variance. Second, it seems reasonable to infer from the correlations of $b t$ and $b w$ values with $e$ that the within replicate variation did not differ simultaneously for temperature and water potential.

In other experiments with other types of within replicate variation it would seem likely that the $b$ sum values would determine the $e$ values. The pattern of correlation discussed here was a product of the responses of the genotypes to the micro-environmental variation encountered. The 
correlations obtained tell more in some ways about the micro-environmental variation than about the genotypes themselves.

\section{Discussion}

It was seen that in the two environmental regimes considered in the experiments the proportion of $V[g]$ to $V[d]$ remained approximately the same. The importance of this relationship lies in the fact that selection procedures will be less complicated due to interaction effects when the proportion of $V[g]$ is low. The possibility that the proportion of $V[g]$ to $V[d]$ would change when a different series of environments or genotypes is tested cannot be discounted.

The behaviour of the genotypes in the two environmental systems, as judged by the relationships between genotypic regression coefficients and germination rates, differed markedly. Genotype by environmental interactions depend on the physical nature of the environments in which the interactions occur and much information is lost when assessments of genotype behaviour are made in a single analysis when there is more than one environmental variable. The physical nature of the environments in field experiments are often unknown. When these environments are assessed by biological means, as they are in the regression analyses under consideration, then no account can be made of non-uniform responses of the genotypes to the different environmental variables.

It has frequently been observed that $[d]$ and $[g]$ values are correlated (Eberhart and Russell, 1966; Perkins and Jinks, 1968). The results demonstrated both correlation or a lack of it between the regression coefficients and germination rates. It was further shown that the correlations could be investigated more deeply by examining individual genotypic values $(y)$ rather than average values $(\bar{y})$. These have revealed some obvious but nevertheless important properties of such correlations which have attracted little comment.

Linear regression coefficients derived over a series of environments will give a positive correlation with $y$ values if these are large enough. A negative correlation will occur with low $y$ values. Hence the correlations between the $b$ values and $y$ values or $\beta$ values and $[d]$ values will depend entirely on the distribution of the environments given. It is imprecise to state a correlation between these parameters when the environments are not defined or when the role of the environmental range in determining the correlation is not acknowledged. It is of great practical importance whether a correlation between $b$ and $y$ or $\bar{y}$ values is present. If the correlation is high, then genotypes which combine a superior performance and a superior stability will rarely be present.

It is useful to consider the relative merits of $\beta$ and $[d]$ values as selection criteria while bearing in mind their interrelationships. If data is available from environments which equate to field environments, then $[d]$ values can form a sound basis for selection. The genotype with the largest $[d]$ value will produce on average the greatest manifestation of the character, e.g. yield under the range of environments likely to occur. Thus regardless of this genotype's $\beta$ value it is the superior genotype and should be selected. Only when a genotype is required in which the yield remains stable in poor environments is it necessary for selection to be made for genotypes with 
low $\beta$ values. Whether such selection is carried out will depend on complex considerations such as the extent of any inferiority in the genotypes' $[d]$ value, to what extent its superior $\beta$ value prevents its yield falling in poor environments, and how important it is to avoid this fall in yield.

When genotypes and environmental ranges are such that several genotypes have equal and superior [d] values, but unequal $\beta$ values, then of these genotypes the one with the lowest $\beta$ value should be selected. However, from published evidence the $\beta$ values associated with high $[d]$ values tend to be higher than the average of the population examined. Thus a successful selection programme for high $[d]$ values will almost inevitably select genotypes with higher than average $\beta$ values. Indeed, genotypes with a high response to nitrogen, for example, may deliberately be selected. Also as variability is exploited over several generations it may become necessary to select genotypes with higher $\beta$ values than those previously employed to gain an increase in $[d]$. For regions of high-fertility selection programmes for high $[d]$ values inevitably select genotypes with high $\beta$ values.

It was shown that $\Delta b$ values were correlated to the remainder terms in the combined analysis. A large non-uniform response of a genotype to temperature and water-potential environments produced a large remainder term in the combined regression. In a regression analysis from field data collected perhaps from various localities and different years the environments contributing to the overall analysis would be of several types. Selection might then be practised on the basis of variation in the genotypic remainder mean squares in addition to $[d]$ or $\beta$ values. For example, Eberhart and Russell (1966) found that the deviations from the regression line formed the greatest proportion of variety by environment interaction. The aim of selection should be, therefore, to develop genotypes showing uniformity in response to environmental effects, i.e. low remainder M.S.'s. Selection for this character would not be complicated since it is not correlated to either $\beta$ or $[d]$ values.

It is interesting to note that Perkins and Jinks (1968) found that for identical genotypes the frequency of significant remainder M.S.'s differed in two sets of environments. A satisfactory explanation for this would be that one set of environments was differing for more physical parameters than the other. It has been argued here that a range of environments which differ for several physical parameters will produce larger remainder M.S.'s in genotypes which have non-uniform responses to different physical parameters. A production of more frequent significant remainder M.S.'s in this manner appears to offer an explanation of the ways in which the greater environmental fluctuation in the one set of experiments might be having their effect. A random increase in experimental error would increase the error M.S.'s as well as the remainder M.S.'s and thus not increase the frequency of significant remainder M.S.'s.

The experiments have shown how remainder M.S.'s which often account for a large proportion of the interaction sums of squares arise. The " model of stability" for this type of analysis (Eberhart and Russell, 1966) is

$$
\Upsilon_{i j}=\mu i+\beta i I j+\delta i j \text {. }
$$

where $\delta i j$ is the deviation from the regression of the $i$ th genotype in the $j$ th environment. In the experiment above the $\delta$ values were correlated to the $\Delta b$ values. More generally $\delta i j$ will be accounted for by the variance 
of the genotypes' response to all of the different environmental variables present. In our particular case this is the $\Delta b$ value since only two responses of the genotype were determined. Therefore, the value $\delta$ is not always analogous to an unpredictable irregularity in the response to the environment as stated by Breese (1969). They are predictable when environmental changes which are not accounted for in the analysis are known. The deviations are not due to developmental noise (Waddington, 1957) or related to the concept of "developmental homeostasis" as discussed by Lerner (1958) since they are determined by changes in genotype response. If these concepts were relevant it would be expected that the remainder terms from the two environments would be correlated, and this relationship was not found.

To take the concept of stability further, it is also seen that microenvironmental variation (e), another aspect of stability, is not random in its nature but determined, at least in part, by predictable responses of the genotype to environmental changes. Whereas $\delta$ values are determined by the changes in genotype responses to environmental variables, $e$ values are determined by the magnitude of genotype responses to environmental variables.

In conclusion, the results presented here demonstrate that regressions techniques to characterise genotype responses to the environment are an oversimplification. Interactions are still occurring which are not identified because biological indices do not give information on the physical nature of the environment. Every genotype has many different responses to environment, each one specific to a particular type of variable, and nonlinear genotype-environmental interactions are explained by these phenomena.

\section{SUMMARY}

1. Genotype by environmental interaction for 45 genotypes of cultivated rape for germination was demonstrated in a range of temperature or waterpotential environments.

2. The responses of the genotypes to the two environmental parameters, when measured by regression analysis, were different.

3. Correlations between regression coefficients and germination rates were found to depend on the response considered and the environment to which the germination rates related.

4. The extent of the deviation from the fitted regression lines shown by the genotypes when the environments were considered together or separated into components depended on the difference in their response to temperature and water potential.

5. Within environment within genotype variation was partially accounted for by the genotype regression coefficients.

6 . The above conclusions were discussed in relation to plant breeding practice.

Acknowledgment.-We wish to thank Mr A. J. McCorquodale for his assistance in purifying and calibrating the Carbowax solutions. 


\section{REFERENCES}

BREESE, E. L. 1969. The measurement and significance of genotype environmental interactions in grasses. Heredity, 24, 27-44.

EBERHART, S. A., AND RUSSELL, W. A. 1966. Stability parameters for comparing varieties. Crop Sci., 6, 36-40.

FINLAY, K. W., AND WILKInson, G. N. 1963. The analysis of adaptation in plant breeding programme. Aust. 7. agric. Res., 14, 742-754.

LERNER, I. M. 1958. The Genetic Basis of Selection. John Wiley \& Sons, Inc., New York.

PERKINS, JEAN M., AND JINKS, J. L. 1968. Environment and genotype environmental components of variability. III. Multiple lines and crosses. Heredity, 23, 339-356.

WADDington, c. H. 1957. The Strategy of the Genes. Allen and Unwin, London.

YATES, F., AND COCHRAN, w. G. 1938. The analysis of groups of experiments. 7 . agric. Sci., Camb., 28, 556-580. 